E5-2005-50

P.E. Zhidkov*

\title{
ON AN INVERSE EIGENVALUE PROBLEM FOR A SEMILINEAR STURM-LIOUVILLE OPERATOR
}

Submitted to «Inverse Problems»

*E-mail: zhidkov@thsun1.jinr.ru 
Жидков П.Е.

Об одной обратной задаче на собственные значения для полулинейного оператора Штурма-Лиувилля

Рассматривается следующая задача: $-u^{\prime \prime}+f(u)=\lambda u, x \in(0,1), u=$ $u(x), u(0)=1, u^{\prime}(0)=u(1)=0$, где $\lambda-$ спектральный параметр. Изучается обратная задача: для заданной части спектра $\lambda_{n} \rightarrow+\infty$ надо найти нечетную $f$. Получено описание всего класса решений этой задачи. Кроме того, показано, что существует не более одной функции $f-$ такой, что некоторая вспомогательная функция не убывает.

Работа выполнена в Лаборатории теоретической физики им. Н.Н. Боголюбова ОИЯИ.

Препринт Объединенного института ядерных исследований. Дубна, 2005

Zhidkov P.E.

E5-2005-50

On an Inverse Eigenvalue Problem for a Semilinear Sturm-Liouville

Operator

The following problem is considered: $-u^{\prime \prime}+f(u)=\lambda u, x \in(0,1), u=$ $u(x), u(0)=1, u^{\prime}(0)=u(1)=0$, where $\lambda$ is a spectral parameter. We study the inverse problem: for a given part of the spectrum $\lambda_{n} \rightarrow+\infty$ we seek odd $f$. We obtain a description of the whole class of solutions of this problem. In addition, we show that there exists at most one function $f$ such that an auxiliary function is nondecreasing.

The investigation has been performed at the Bogoliubov Laboratory of Theoretical Physics, JINR. 


\section{INTRODUCTION}

Nonlinear second-order elliptic eigenvalue problems have been intensively discussed in the mathematical literature during several last decades. At the same time, results on inverse problems for these equations are in fact unknown. As for inverse problems for linear self-adjoint second-order ordinary differential equations, now the classical Borg uniqueness theorem is well-known (see, for example, [1]): in fact, for given two sequences $\left\{\lambda_{n}^{1}\right\}_{n=0,1,2, \ldots}$ and $\left\{\lambda_{n}^{2}\right\}_{n=0,1,2, \ldots}$ there exists at most one real-valued potential in the operator so that these sequences are the spectrums of this operator taken with two different sets of standard boundary conditions.

In the present paper, we consider an inverse problem for the simplest nonlinear Sturm-Liouville-type operator in a bounded interval. In fact, we study the equation

$$
-u^{\prime \prime}+f(u)=\lambda u, \quad u=u(x), x \in(0,1),
$$

supplied with boundary conditions

$$
u(0)=1, u^{\prime}(0)=u(1)=0 .
$$

Here $\lambda$ is a spectral parameter. Our result also holds for the same equation with the boundary conditions

$$
u(0)=1, u^{\prime}(0)=u^{\prime}(1)=0
$$

our method applies without modifications except the simplest ones. Hereafter, all the quantities are real. We consider the inverse problem: given some sequence of eigenvalues $\lambda_{n} \rightarrow+\infty$ (with the unknown corresponding eigenfunctions $u_{n}$ ), we seek the corresponding nonlinearity $f(u)$. Note that to each eigenvalue there corresponds a unique eigenfunction because the boundary conditions contain partly the Cauchy data $u(0)=1, u^{\prime}(0)=0$. Set $F(u)=2 \int_{0}^{u} f(t) d t$. As readers will see further, for any function $f$ odd and continuously differentiable in $\mathbb{R}$ any eigenfunction $u$ of problem (1)-(2) taken with this function $f$ satisfies $|u(x)| \leqslant 1$. So, in what follows, we use the following assumptions on the nonlinearity $f$.

(f) Let $f(u)$ be a continuously differentiable odd function of $u \in[-1,1]$.

As is shown in [2,3], unlike the statement of the problem in the linear case, it is natural to consider a nonlinear eigenvalue problem with an additional normalization condition as $u(0)=1$ or similar. An explanation is the following. Problem 
(1)-(2) with a given odd nondecreasing nonlinearity $f$ has an infinite sequence of eigenvalues $\lambda_{n}$ with the corresponding eigenfunctions $u_{n}(n=0,1,2, \ldots)$ such that each $n$th eigenfunction $u_{n}$ has precisely $n$ zeros in $(0,1)$, there are no any other eigenvalues and eigenfunctions and the sequence $\left\{u_{n}\right\}_{n=0,1,2, \ldots}$ of all eigenfunctions is a basis (in addition, a Riesz basis) in $L_{2}(0,1)$. So, the problem that includes the normalization condition $u(0)=1$ has properties quite similar to those of a linear self-adjoint second-order problem. At the same time, if one excludes the normalization condition from the statement of the problem, then the set of all eigenfunctions becomes too wide.

Let $g(u)=(F(u)-F(1)) /\left(1-u^{2}\right)$. Our result here is the following.

Theorem. Let $\left\{\lambda_{n}\right\}_{n=1,2,3, \ldots}$ be a sequence of real numbers such that $\lambda_{n} \rightarrow$ $+\infty$ as $n \rightarrow \infty$. Then

(a) if there exists an increasing sequence of positive integers $m_{n} \rightarrow \infty$ such that each $\lambda_{n}=\left[\left(\pi\left(2 m_{n}+1\right)\right) / 2\right]^{2}$, then there exists a unique nonlinearity $f$ obeying hypothesis (f) such that each $\lambda_{n}$ is an eigenvalue of problem (1)-(2) taken with this function $f$ and this function $f$ is identically equal to 0 in $[-1,1]$;

(b) There exists at most one solution $f_{1}$ (or $\left.f_{2}\right)$ of our inverse problem satisfying hypothesis $(f)$ with the nondecreasing corresponding function $g=g_{1}(u)$ (resp., with the nonincreasing corresponding function $g=g_{2}(u)$ );

(c) For any function $h$ continuous in $\mathbb{R}$ and arbitrary two solutions $f_{1}$ and $f_{2}$ of the inverse problem with the corresponding functions $g=g_{1}(u)$ and $g=g_{2}(u)$ one has

$$
\int_{0}^{1} h\left(g_{1}(u)\right)\left(1-u^{2}\right)^{-1 / 2} d u=\int_{0}^{1} h\left(g_{2}(u)\right)\left(1-u^{2}\right)^{-1 / 2} d u \text {. }
$$

Remark 1. A function $g$ uniquely determines the corresponding function $f$.

\section{PROOF OF THE THEOREM}

Below we summarize results for the direct problem (1)-(2) (with a given $f$ ) proved in fact in $[2,3]$.

Proposition. Let $f$ be a function continuously differentiable and odd in $\mathbb{R}$. Then

(a) for any integer $n \geqslant 0$ problem (1)-(2) has an eigenvalue $\lambda_{n}$ with the corresponding eigenfunction $u_{n}(x)$ such that $u_{n}$ possesses precisely $n$ zeros in $(0,1)$;

(b) for each $n$ and an eigenfunction $u$ that possesses precisely $n$ zeros in $(0,1)$ the zeros of $u$ in $(0,1)$ are precisely the points $x_{i}^{n}=\frac{1}{2 n+1}+\frac{2 i}{2 n+1}, i=$ $0,1, \ldots, n-1$; 
(c) any eigenfunction has a finite number of zeros in $(0,1)$;

(d) $u^{\prime}(x)<0$ in $\left(0, x_{1}^{n}\right]$ for an arbitrary eigenfunction u possessing precisely $n$ zeros in $(0,1)$;

(e) $|u(x)| \leqslant 1$ for all $x \in[0,1]$ and for an arbitrary eigenfunction $u$.

Let us return to our inverse problem. Let $\left\{\lambda_{n}\right\}_{n=1,2,3, \ldots}$ be a sequence of real numbers going to $+\infty$ as $n \rightarrow \infty$ and let $f_{1}$ and $f_{2}$ be two solutions of our inverse problem that obey hypothesis (f). Denote by $\mu_{n}=([\pi(2 n+1)] / 2)^{2}, n=$ $0,1,2, \ldots$, the set of all eigenvalues of the linear problem

$$
-w^{\prime \prime}=\mu w, \quad w=w(x), x \in(0,1), w^{\prime}(0)=w(1)=0 .
$$

Denote also by $k_{n}^{i}$ the number of zeros in $(0,1)$ of the solution $u_{n}^{i}$ of problem (1)-(2) taken with $f=f_{i}$ and with $\lambda=\lambda_{n}$. Then, by the standard comparison theorem, $k_{n}^{i} \rightarrow+\infty$ as $n \rightarrow \infty$ and there exists $D>0$ such that

$$
\left|\lambda_{n}-\mu_{k_{n}^{i}}\right| \leq D, \quad n=1,2,3, \ldots, i=1,2
$$

(because the $n$th eigenfunction $w_{n}$ of the linear problem (4) has precisely $n$ zeros in $(0,1)$ ). Therefore, $\left|\mu_{k_{n}^{1}}-\mu_{k_{n}^{2}}\right| \leqslant 2 D$ for all $n$. Hence, we have $k_{n}^{1}=k_{n}^{2}$ for all sufficiently large $n$ because $\mu_{n+1}-\mu_{n} \rightarrow+\infty$ as $n \rightarrow \infty$. Let $k_{n}=k_{n}^{1}$. We have proved following result.

Lemma 1. For all sufficiently large $n$ the eigenfunctions $u_{n}^{1}$ and $u_{n}^{2}$ have the same number of zeros $k_{n}$ in $(0,1)$.

Denoting now by $x_{1}^{n, i}$ the smallest positive zero of $u_{n}^{i}$, we get $x_{1}^{n, 1}=x_{1}^{n, 2}$ for all sufficiently large $n$. Without the loss of generality, we accept that this is valid for all $n$. Set $x_{n}=x_{1}^{n, 1}$.

Now, we apply the solvability of Eq. (1) by quadratures. Multiply it by $2 u^{\prime}(x)$ and integrate the result from 0 to $x$. Then, we obtain the identity

$$
\left[u^{\prime}(x)\right]^{2}+F(1)-F(u(x))+\lambda u^{2}(x)-\lambda \equiv 0, \quad x \in[0,1] .
$$

Using property (d) and denoting by $x_{n}(u)$ the function inverse to $u_{n}(x)$ in $\left[0, x_{n}\right]$, we derive from (5):

$$
x_{n}^{\prime}(u)=-\frac{1}{\sqrt{\lambda_{n}-\lambda_{n} u^{2}+F(u)-F(1)}}, \quad u \in[0,1),
$$

and

$$
x_{n}=\int_{0}^{1} \frac{d u}{\sqrt{\lambda_{n}-\lambda_{n} u^{2}+F(u)-F(1)}}, \quad n=1,2,3, \ldots
$$

Note that $u=1$ is the point of singularity for the expression in the right-hand side of (6). However, there exists $C>0$ such that

$$
\lambda_{n}-\lambda_{n} u^{2}+F(u)-F(1) \geq C(1-u)
$$


for all $u \in[0,1)$ and for all sufficiently large $n$ so that the improper integral in (6) is absolutely converging for all sufficiently large $n$. In addition, in view of (7), $u=1$ is the only point of singularity in (6) for all sufficiently large $n$.

Let us prove statement (a) of the theorem. Clearly, $f \equiv 0$ is a solution of the inverse problem. Let $f$ be an arbitrary its solution satisfying hypothesis (f). We have from (6) using Taylor expansions:

$$
\begin{gathered}
\lambda_{n}^{-1 / 2} \int_{0}^{1}\left(1-u^{2}\right)^{-1 / 2} d u= \\
=\lambda_{n}^{-1 / 2} \int_{0}^{1}\left(1-u^{2}\right)^{-1 / 2}\left[1-\frac{1}{2} \lambda_{n}^{-1} g(u)+\frac{3}{8} \theta \lambda_{n}^{-2}[g(u)]^{2}\right] d u=x_{n}
\end{gathered}
$$

where $\theta=\theta(n, u) \in(0,1)$. Hence, $\int_{0}^{1}\left(1-u^{2}\right)^{-1 / 2} g(u) d u=0$ and thus, $g(u) \equiv 0$ which completes the proof of statement (a).

Let again $f_{1}$ and $f_{2}$ be arbitrary two solutions of our inverse problem satisfying hypothesis (f) with the corresponding functions $F_{i}$ and $g_{i}, i=1,2$.

Lemma 2. One has $\int_{0}^{1}\left(1-u^{2}\right)^{-1 / 2}\left[g_{1}(u)\right]^{l} d u=\int_{0}^{1}\left(1-u^{2}\right)^{-1 / 2}\left[g_{2}(u)\right]^{l} d u$ for all $l=0,1,2, \ldots$ (it is easy to see that the improper integrals here are absolutely converging).

Proof. Take a sufficiently large $n$ and consider the expression

$$
\begin{aligned}
& x_{n}=\int_{0}^{1}\left(\lambda_{n}-\lambda_{n} u^{2}+F_{i}(u)-F_{i}(1)\right)^{-1 / 2} d u= \\
& =\lambda_{n}^{-1 / 2} \int_{0}^{1}\left(1-u^{2}\right)^{-1 / 2}\left(1+\lambda_{n}^{-1} g_{i}(u)\right)^{-1 / 2} d u .
\end{aligned}
$$

For any $\epsilon>0$ the expression $\left|\lambda_{n}^{-1} g_{i}(u)\right|$ is smaller than $\epsilon$ for all sufficiently large $n$ and it is continuous in $u \in[0,1]$ (at the point $u=1$, there exists a limit of $g_{i}(u)$ as $\left.u \rightarrow 1-0\right)$. So, for all sufficiently large $n$, we can expand the expression in the right-hand side of (8) into a Taylor's series obtaining

$$
x_{n}=\lambda_{n}^{-1 / 2} \sum_{l=0}^{\infty} \lambda_{n}^{-l} A_{l} \int_{0}^{1}\left(1-u^{2}\right)^{-1 / 2}\left[g_{i}(u)\right]^{l} d u, i=1,2,
$$


so that

$$
\sum_{l=0}^{\infty} \lambda_{n}^{-l} A_{l} \int_{0}^{1}\left(1-u^{2}\right)^{-1 / 2}\left[g_{1}(u)\right]^{l} d u=\sum_{l=0}^{\infty} \lambda_{n}^{-l} A_{l} \int_{0}^{1}\left(1-u^{2}\right)^{-1 / 2}\left[g_{2}(u)\right]^{l} d u
$$

for all sufficiently large $n$ where

$$
A_{0}=1 \text { and } A_{l}=\frac{(-1 / 2)(-1 / 2-1)(-1 / 2-2) \ldots(-1 / 2-l+1)}{l !}
$$

for $l=1,2,3, \ldots$ so that these coefficients do not depend on $i, n$ and $u$ and $\left|A_{l}\right| \leqslant 1$ for all $l$. Since $\lambda_{n} \rightarrow+\infty$ as $n \rightarrow \infty$, the relations above yield

$$
\int_{0}^{1}\left(1-u^{2}\right)^{-1 / 2}\left[g_{1}(u)\right]^{l} d u=\int_{0}^{1}\left(1-u^{2}\right)^{-1 / 2}\left[g_{2}(u)\right]^{l} d u, \quad l=0,1,2, \ldots,
$$

and lemma 2 is proved. $\square$

Lemma 3. For any function $h(s)$ continuous in $\mathbb{R}$ one has

$$
\int_{0}^{1}\left(1-u^{2}\right)^{-1 / 2} h\left(g_{1}(u)\right) d u=\int_{0}^{1}\left(1-u^{2}\right)^{-1 / 2} h\left(g_{2}(u)\right) d u .
$$

Proof. Denote $M=\sup _{i=1,2, u \in[0,1)}\left|g_{i}(u)\right|+1$. Due to lemma 2 and the linearity, for any polynomial $h(s)$ of the kind $h(s)=\sum_{m=0}^{N} a_{m} s^{m}$ with real coefficients $a_{m}$ the statement of lemma 3 holds. In the general case, it follows by approximations of $h(s)$ uniform with respect to $u \in[-M, M]$ by polynomial functions. $\square$

Remark 2. With lemma 3, we have proved statement (c) of our theorem.

Remark 3. Of course, equality (3) is a necessary condition for a function $f_{2}$ to be a solution of the inverse problem if $f_{1}$ is known. However, in view of (6) and (8), this condition is «almost» sufficient.

Let us prove statement (b) of the theorem. On the contrary, suppose the existence of two solutions $f_{1}$ and $f_{2}$ of the inverse problem such that the corresponding functions $g_{1}$ and $g_{2}$ are nondecreasing (for nonincreasing functions $g_{i}$, the proof can be made by the complete analogy). Take an arbitrary continuous $h$ and make in the integrals in (3) the change of the independent variable by setting $z=\arcsin u$. Then, we obtain: $I_{1}(h)=I_{2}(h)$ where $I_{i}(h)=\int_{0}^{\pi / 2} h\left(p_{i}(z)\right) d z$ and $p_{i}(z)=g_{i}(\sin z)$. 
First of all, it is easy to see that $p_{1}(0)=p_{2}(0)$ and $p_{1}(\pi / 2)=p_{2}(\pi / 2)$ by lemma 3. Denote $p_{1}(0)=A$ and $p_{1}(\pi / 2)=B$. By $S$, denote the set of all points $p \in[A, B]$ such that, for each $i=1,2$, the preimage $p_{i}^{-1}(p)$ consists of a unique point $z_{i}=z_{i}(p)$ and $\left.p_{i}^{\prime}(z)\right|_{z=z_{i}(p)} \neq 0, i=1,2$. It is easy to see that the set $S$ is dense in $[A, B]$. First, let $p \in S$. Take for the function $h=h(s)$ the characteristic function of the interval $[A, p]$ equal to 1 if $s \in[A, p]$ and 0 otherwise. It is easy to see that equality (3) still holds for this function $h$. This equality immediately implies that $z_{1}(p)=z_{2}(p)$.

Now, consider the case when $p \in[A, B]$ and $p \notin S$. Then, there exist two sequences $p_{2}^{s} \searrow p$ and $p_{1}^{s} \nearrow p$ such that $p_{i}^{s} \in S, i=1,2$. Hence, by the proved above, $z_{1}\left(p_{i}^{s}\right)=z_{2}\left(p_{i}^{s}\right), i=1,2$. Therefore, $z_{i}\left(p_{1}^{s}\right) \rightarrow \bar{z}, z_{i}\left(p_{2}^{s}\right) \rightarrow \overline{\bar{z}}$ as $s \rightarrow \infty$ (the limits $\bar{z}$ and $\overline{\bar{z}}$ do not depend on $i$ ) and $p_{i}^{-1}(p)=[\bar{z}, \overline{\bar{z}}]$ so that $\left\{z: p_{1}(z)=p\right\}=\left\{z: p_{2}(z)=p\right\}$. So, we have proved that $p_{1}(z)=p_{2}(z)$ for all $z \in[0, \pi / 2]$ and statement (b) is proved. Our proof of the theorem is complete.

Example of non-uniqueness. Obviously, for any $h$ one has

$$
\int_{0}^{\pi / 2} h(f(\sin 2 z)) d z=\frac{1}{2} \int_{0}^{\pi} h(f(\sin z)) d z=\int_{0}^{\pi / 2} h(f(\sin z)) d z .
$$

According to (6) and (8), there exists a sequence of eigenvalues $\lambda_{n} \rightarrow+\infty$ of problem (1)-(2) taken with each of these two nonlinearities $f(u)$ and $f(\sin (2 \arcsin u))$ where $u=\sin z$.

\section{REFERENCES}

1. Levitan B. M., Sargsyan I. S. Sturm-Liouville and Dirac Operators. M.: Nauka, 1988 (in Russian).

2. Zhidkov P. E. Riesz Basis Property of the System of Eigenfunctions for a Nonlinear Problem of Sturm-Liouville Type. // Sbornik: Mathematics. 2000. V.191, No. 3. P. 359-368.

3. Zhidkov P.E. Basis Properties of Eigenfunctions of Nonlinear Sturm-Liouville Problems // Electron. J. Diff. Eqns. 2000. V. 2000, No. 28. P. 1-13.

Received on April 21, 2005. 
Корректор T. Е. Попеко

Подписано в печать 13.05.2005.

Формат $60 \times 90 / 16$. Бумага офсетная. Печать офсетная.

Усл. печ. л. 0,68. Уч.-изд. л. 0,96. Тираж 315 экз. Заказ № 54877.

Издательский отдел Объединенного института ядерных исследований 141980, г. Дубна, Московская обл., ул. Жолио-Кюри, 6.

E-mail: publish@pds.jinr.ru www.jinr.ru/publish/ 